\title{
Organizational and technological risks during the formation of clusters in megapolis
}

\author{
Mikhail Semenenko, and Stepan Dral*, \\ Moscow State University of Civil Engineering, 129377, Moscow, Russia
}

\begin{abstract}
The paper presents the characteristics of the risks in the formation of residential clusters in a megalopolis. The main goal of the model of clusters of integrated urban development is the high efficiency of its functioning and implementation. Such a development model allows simultaneously realizing many goals: economic, social, technological, and also allows monitoring various construction parameters and controlling them at all stages of construction production. The paper gives a definition of risks in construction, a classification of the risks of cluster formation, and also provides methods for their assessment and control. In addition to theoretical data, the paper provides examples of situations both in Russia and abroad.
\end{abstract}

\section{Introduction}

The formation of residential clusters is characterized by many types of risk factors that arise at different stages. Construction risks arise at the stage of construction of facilities, causing violations of technology and terms of construction. The types of risks can be divided into external and internal ones. The main external ones include natural, economic and administrative risks. Internal risks are divided into resource risks and partner risks, including risks of managing flow processes and risks of interaction between the subjects of the supply system, they are called organizational technological risks of construction. As a rule, external risks are not affected by the participants in the construction project, therefore, less attention is paid to their elimination [1].

Due to the scope of work and the total number of participants in the formation of residential clusters, internal risks are also often spontaneous in nature. They are unpredictable, and their consequences are irreversible. Therefore, the terms of construction are usually postponed, or the result of construction itself is of a lower quality than it was projected.

\section{Materials and methods}

\footnotetext{
*Corresponding author: stepandra149@gmail.com
} 
Nowadays, the concept of risk has received more than one version of interpretation, from which it follows that, first of all, it is necessary to determine which risks will have to be dealt with. Summarizing the potential risk under one of the definitions in the table below, one can understand its potential danger, as well as the possible benefit that can be obtained with the correct risk management strategy [2].

Risk management is a system for making and implementing decisions designed to minimize the negative consequences of the implementation of risks on the organization's activities [3].

Often a matrix of subsystems is used as a risk management tool, which makes it possible to clearly define functional relationships for optimal management [4].

Table 1. Risk classification

\begin{tabular}{|c|c|c|}
\hline Concept & Idea & $\begin{array}{l}\text { Main type of risk Areas of life application } \\
\text { management }\end{array}$ \\
\hline
\end{tabular}

Risk as a Risk carries only Reducing the Basics of life safety, danger, a threat the danger likelihood and (or) OHS, insurance (from associated with the consequences of the client's point of onset of adverse adverse events view) consequences Risk as an Risk assumes the
opportunity, possibility of not chance only negative consequences, but also the possibility of success, gain. In this case, usually, the higher the risk, the higher the potential gain.

Risk as an Risk lies in the uncertainty of possible deviation a result, a of the actual possibility of results from the its deviation expected (target). from a goal
Maximizing benefits while limiting losses

Reducing the scatter (variance), the likelihood of large deviations of the actual results from the expected.
Risk management when working in financial markets (financial risk management) Gambling
Equipment (precision manufacturing of parts), quality management, weapons systems (target detection, hitting the target)

\begin{tabular}{|c|c|c|c|c|c|c|c|c|c|}
\hline $\begin{array}{l}\text { Management and } \\
\text { production units }\end{array}$ & 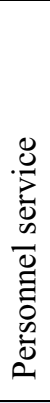 & 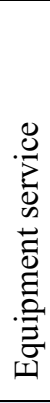 & 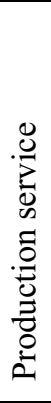 & 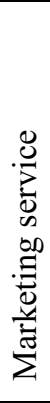 & 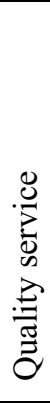 & 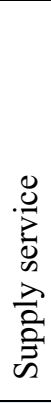 & 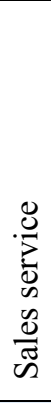 & 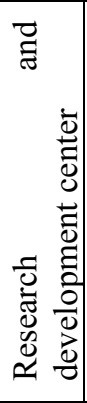 & 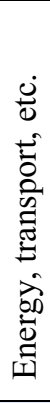 \\
\hline \multicolumn{10}{|l|}{ Labor organization } \\
\hline $\begin{array}{l}\text { Organization of the } \\
\text { use of tools }\end{array}$ & & & & & & & & & \\
\hline
\end{tabular}




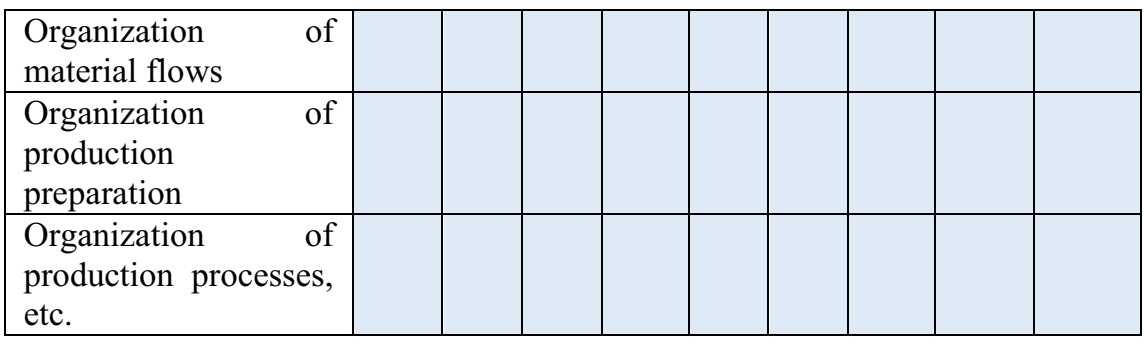

Fig. 1. Example of a subsystem matrix

In modern construction, risk management and assessment are of great importance. Therefore, many ways and methods of their prevention and consideration have been developed. With proper risk management, it is necessary to take into account many factors and follow certain principles: make conscious risk decisions and develop a strategy to prevent risks in unsuccessful cases in advance, compare the levels of risks and the company's capabilities, and understand the economic feasibility of such decisions.

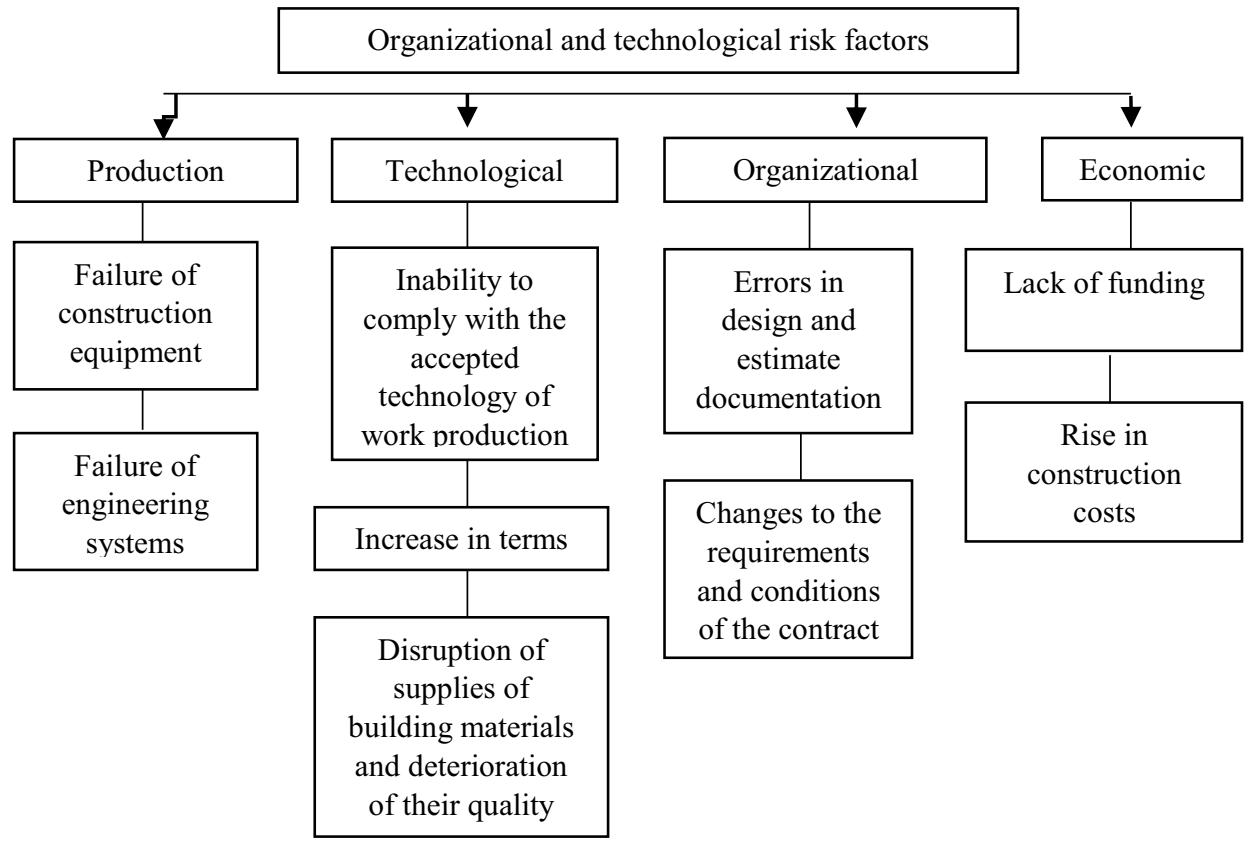

Fig. 2. Organizational and technological risk factors and their consequences

All of the above factors are closely related to each other and can act both as the main causes of construction problems and as secondary factors of some other problem. So, for example, low financing can affect the supply of materials, or, on the contrary, an increase in the cost of materials can affect an increase in the estimated cost, which will lead to financial insecurity [5].

According to statistics, up to $50 \%$ of the financial losses of construction organizations fall on the labor resources used, $20 \%$ on organizational and technological reasons, $26-28 \%$ on external factors, and $2-4 \%$ on the used building materials.

A cluster is a territorial entity within a megalopolis, which is a relatively autonomous unit and provides its residents with a full set of urban functions [6]. Let's consider this concept using the example of Moscow - residential clusters, as a rule, include transport infrastructure (organization of internal roads, ramps, ensuring transport accessibility of 
residents), construction of various municipal services, such as kindergartens, schools, and sometimes clinics. Also, upon completion of the formation of the cluster, it is filled with a commercial sector, which provides a variety of leisure activities and shops for buying everything that residents may need.

Since a residential cluster in its essence is an investment and construction project (ICP), then the risks in its formation also include all the main risks that an ICP has in the process of formation and implementation.

When considering organizational and technological risks separately, there are many situations that will lead to their occurrence. Nowadays, a fairly common phenomenon is the dishonesty of people responsible for the supply of raw materials to construction sites. Thus, in case of negligence or when trying to profit from their position, when checking the compliance with the requirements of the project, violations of technologies for the construction of load-bearing structures or a decrease in the bearing strength class of materials from which they are made will be found. In order to avoid such situations, during the development of projects and the construction of objects, an examination is always carried out, which should identify such violations both in finished projects and objects, and at the stage of their creation or construction. There is also widespread dishonesty of construction workers, which entails delays in time due to productivity lower than that projectedin a work schedule.

Many risks can be avoided by developing new, more modern construction production technologies and using modern materials. Also, computerization has a positive effect on reducing risks in construction, thanks to which the design process, the relationship between the organizations involved in the construction and the construction process itself are greatly simplified, but it is not always possible to develop a new construction technology, or to apply safer, but more expensive building materials.

The worst of the construction risks are design errors that were not noticed during the examination. Such situations, depending on the scale, can have consequences of varying severity, from economic damage to the death of people.

There are many examples of major design errors. An example is the tragedy that occurred in Moscow on February 14, 2004. Then, as a result of a spontaneous decision to amend the project and the inconsistency of the two design companies, the dome of the Transvaal Park sports and entertainment complex collapsed.

In addition to the designers' mistakes, tragedies happen through the fault of the owners of the buildings. The collapse of the Basmanny Market in Moscow on February 23, 2006, was an example of a fatal violation of the building's rules of operation. The collapse of the building occurred due to corrosion of the load-bearing elements, and also, according to some versions, due to exceeding the permissible load on the mezzanine floor.

Technological disruptions during construction are also very insidious and long-term risks. Such mistakes may not manifest themselves immediately. Tens of years may pass before improperly reinforced structures collapse under the design load that they had to withstand.

In 2003, a tornado in the United States destroyed the Kinzua Bridge built in 1882. The cause of the collapse was a technological error in strengthening the supports. Due to the increase in the consumption of coal, which was transported by railroad trains across this bridge, it was necessary to replace the iron supporting structures with steel ones, but the thickness of the base plate at the steel portals of the bridge was greater than the thickness of the similar plate at the iron supports. To save time, the workers decided not to replace the reinforced concrete foundations of the portals, but to fix them by lengthening the old anchor bolts. Due to corrosion, they could not withstand the wind load and burst, thereby collapsing the bridge, which was no longer used for commercial purposes by the $21 \mathrm{st}$ century [7]. 
In recent years, in Russia and the CIS countries, we have also been able to observe situations of workers' strikes due to delays in wages by the employer, which is a consequence of economic mistakes of the management and underfunding of construction. Such errors can lead to a halt in construction, which is unacceptable in the case of cluster development. This risk is difficult to assess and foresee due to the distribution of tasks for the formation of clusters among contractors. This approach makes it almost impossible to control the wages of workers, since these are responsibilities of the contractor, who does not report this to the project investors.

The COVID-19 pandemic was an excellent example of external risks, which led to the bankruptcy of some companies and the need to stop construction. Such risks cannot be foreseen, and it is also impossible to reasonably assess their danger, as it happened with coronavirus infection. It also increased the costs of development and construction of facilities due to the need for regular monitoring of morbidity and the provision of remote work for some organizations.

To prevent more damage from the spreading virus, construction sites have begun to take countermeasures to ensure the safety of construction workers and meet deadlines. Until the moment of victory over COVID-19, all personnel must adhere to the mask regime at all construction sites. In addition to the use of personal protective equipment, all workers have their body temperature measured to identify potential carriers of infection. All workers who show symptoms of SARS are not allowed to the workplace without a negative PCR test result.

\section{Results and discussion}

Timely and correct management of risks in the construction industry helps prevent many negative events that range from small losses for the contractor to tragedies that led to the death of people. To prevent such situations, special methods of risk assessment are used, which subsequently make it possible to obtain a way to prevent them, or to reduce damage.

There are 4 main methods for assessing the level of risk:

1. Expert methods. They are a survey of qualified specialists and subsequent processing of the result with the adoption of the most optimal decision based on many factors, for example, the authority and experience of an expert. As a rule, they are used in the absence or lack of data for other methods.

2. Analog methods. They are used to assess the risk of the largest and most repetitive operations.

3. Statistical methods. They require a lot of data to compile a representative sample from which a quantitative idea of the level of risk will be obtained.

4. Calculation and analytical methods. They are a set of criteria calculated according to certain algorithms and allowing a qualitative assessment of risks [8].

Let's consider each of the methods in more detail.

\subsection{Expert method}

The first step in this method may be to assess the importance of each quality that experts possess. For this, a register of necessary qualities is compiled, and then, using the method of paired comparisons, a matrix of preferences is compiled. According to the estimates obtained in the preference matrix, the weight of the significance of each of the qualities is calculated, based on which it is then possible to determine the level of the expert's competence.

For this, experts are selected who assess the level of development of a particular quality in experts who assess risks. The assessment is divided into several levels, each of which is 
assigned a numerical value. Then a general assessment of the competence of each expert is determined according to the formula:

$$
A_{k}=\sum_{j=1}^{m} V_{i} \cdot a_{i j k}
$$

where

$a_{i j k}$ - assessment of the $i$-th quality of the $k$-th expert assessing risks given by the $j$-th expert.

$V_{i}$ - weight of significance of the $i$-th quality.

Further, conditions are drawn up for the distribution of experts according to the level of competence, and the results of the calculation are systematized.

At the next stage, each expert sets the probability of occurrence $P_{i}$ and the value of losses $l_{q}$ for the risks that need to be assessed.

The risk index is calculated by the formula:

$$
x_{i j}=P_{i} \cdot l_{q}
$$

The final risk index is calculated by multiplying the risk index put down by the expert and his level of competence.

Then, using statistical methods, the degree of consistency of expert assessments on the $i$-th risk is calculated [9].

\subsection{Analog methods}

These methods find their application in the assessment of investment, credit and other economic risks. The essence of these methods is to compare the risk to be assessed with situations that have had similar operations and transactions. Thus, it is possible to predict possible investment mistakes made earlier.

\subsection{Statistical methods}

Using statistical methods, it is possible to quantify the level of risk and determine the likelihood of economic risks. With the help of statistics, it is also possible to determine the likelihood of risks due to various surveys that contain a sufficient amount of data for this kind of results processing [10]. Geodetic surveys can be studied statistically.

There are many statistical methods, but one of the most applicable in construction is the probability estimation method. It is divided into 2 sub-methods.

Expected average calculation - determining the numerical value of a contingency, which is defined as the weighted average of all possible outcomes, where the probability of those outcomes is applied as the frequency or weight of the corresponding value.

The variability of a possible outcome is the level of deviation of the expected value from the average one. To do this, two criteria are applied: standard deviation and variance.

Variance is the weighted average of the squares of the deviations of the actual results from the expected average.

$$
\sigma^{2}=\frac{\sum(x-\bar{x})^{2}}{n}
$$

where $\sigma^{2}$ - variance;

$x$ - expected value for each observation;

$\bar{x}$-expected average value;

$n$ - number of cases.

Mean square deviation is calculated by the formula: 


$$
\sigma=\sqrt{\sigma^{2}}
$$

The coefficient of variation is used for the analysis:

$$
V= \pm \frac{\sigma}{x} \cdot 100 \%
$$

Where $V$ - coefficient of variation, $\%$

\subsection{Calculation and analytical methods}

They are widely used in assessing economic risks and are based on mathematical modeling. There are 4 methods related to calculation and analytical methods:

1. Sensitivity analysis. The essence of the method is to demonstrate the change in the total indicator when the initial parameters change.

2. Method of adjusting the discount rate taking into account risk. Based on a change in the basic discount rate. Regulation occurs by adding the value of the required risk premium.

3. Method of reliable equivalents. It is used to adjust the estimated values of cash flows by multiplying them by special confidence factors

4. Scenario method. It consists in drawing up "scenarios" according to which events will develop in the future. Allows predicting possible risks long before their manifestation. The method is applicable in construction in order to take into account possible external risks.

\section{Conclusions}

The construction industry is an area with a huge number of risks and their factors, especially when it comes to the development of residential clusters, the formation of which requires huge resources. With such a volume of construction, it is necessary to provide for many subtleties and nuances that vary depending on the place of construction, residents of the built-up area, material and technical base, labor resources and many other factors, each of which carries certain risks and is capable of provoking problems in related risk categories. It is for this reason that it is necessary in construction to pay great attention to risk management and the ability to compensate for losses and negative events caused by unpredictable events such as pandemics or natural disasters.

\section{References}

1. A.I. Shnyrova, Modern methods of risk assessment in construction (2015).

2. S.B. Bogoyavlenskiy, Risk management in socio-economic systems, Textbook (2005).

3. GOST R 56275-2014 Risk management. Guide to good practice for project risk management

4. M.F. Semenenko, Formation of a management system for organizational models for the implementation of quarterly development projects.

5. D.I. Vinogradova and R.G. Abakumov, Factors of the organizational and technological risk environment of investment and construction activities (2016).

6. M.F. Semenenko, Organizational and technological reliability of the formation of a cluster of urban residential development.

7. L. Jeffrey, Fall of the Eighth Wonder (The Kinzua Bridge, 2009). 
8. T.Kh. Ablyazov and E.B. Aleksandrova, Risks of the implementation of investment and construction projects in the context of the formation of the digital economy (2018).

9. N.A. Lapteva and T.F. Morozova, Assessment of risks in the implementation of an investment and construction project using the example of a business center, Magazine of civil engineering, 2 (2011).

10. V.O. Makarova and E.A. Arlamov, Methodological approaches to the assessment of construction risks (2017). 